\title{
Valence Band of Cubic Semiconductors from Viewpoint of Clifford Algebra
}

\author{
A. DARGYS* \\ Semiconductor Physics Institute, A. Goštauto 11, LT-01108 Vilnius, Lithuania
}

(Received March 6, 2009; in final form April 16, 2009)

\begin{abstract}
In this paper the properties of semiconductors having cubic symmetry are considered in a real multidimensional Euclidean space within the formalism of multivector Clifford algebra rather than, usually used for this purpose, complex Hilbert space. In particular, it is demonstrated how the valence band energy spectrum and spin properties may be calculated within $C l_{5}$ Clifford algebra and $\mathrm{SO}(5)$ symmetry group related with it.
\end{abstract}

PACS numbers: 71.15.-m, 72.25.Dc, 75.10.Jm, 85.75.-d

\section{Introduction}

Clifford algebras are the vector space algebras with quantum mechanical spins incorporated within one mathematical system. It is believed that these algebras provide the most coherent mathematical language for physicists $[1,2]$. Coordinate geometry, vector analysis, complex analysis, tensor analysis, Lie groups, differentials forms, spinor analysis, twistors etc. can be incorporated within the Clifford algebra formalism. A part of the Clifford algebra adapted to physics, in particular to describe the Newtonian and Minkowski timespace, was named the geometric algebra [3-5]. Despite high expectations for the Clifford algebras to become the basic computational tool in physics, this has not happened as yet. Partly this can be explained by the fact that math curriculum is not well matched to needs of the physics. Secondly, the geometric algebra is relatively young mathematical physics discipline and, according to the arguments of Dyson and Hestenes, the passionate advocate of the geometric algebra, "the innovations in mathematical physics are almost always unfashionable when they are introduced", so "it takes 50 to 100 years for them to achieve general recognition". In Hestenes popular review [3] the reader can find more references as well as websites on the Clifford algebra.

In this paper the Clifford algebra $C l_{5}$ is applied to analysis of hole spectrum and spin properties of the valence band of cubic semiconductors. The typical representatives are $\mathrm{A}_{3} \mathrm{~B}_{5}$ compounds, the valence band of which is described by the Luttinger-Kohn Hamiltonian [6]. In papers [7-9] the hole spin properties were analyzed in a standard manner within the Hilbert space, either using unitary matrices, or commutators to describe nontrivial

\footnotetext{
* e-mail: dargys@pfi.lt
}

free-hole spin precession properties. Here the problem will be treated within Clifford algebra formalism that is related to $\mathrm{SO}(5)$ symmetry group. It is shown that the diagonalization of the Hamiltonian can be viewed as a rotation of the Clifford vector in the five dimensional real $\mathbb{R}^{5}$ Euclidean rather than complex Hilbert space. Since the considered subject is relatively uncommon to solid-state physicists, the needed properties of $\mathrm{Cl}_{5}$ and terminology of the Clifford algebra are introduced at first. Then the valence band properties are analyzed from the $C l_{5}$ algebra point of view. Spin properties of conduction band electrons in cubic semiconductors were recently analyzed within the Clifford algebra formalism in paper [10].

\section{Relevant properties of $C l_{5}$ Clifford algebra}

\subsection{General properties}

$C l_{5}$ is made up of $2^{5}=32$ elements usually denoted by bold $e$ :

- one scalar: $\boldsymbol{e}_{0}$

- five vectors: $e_{1}, e_{2}, e_{3}, e_{4}, e_{5}$;

- ten bivectors: $e_{12}, e_{13}, e_{14}, e_{15}, e_{23}, e_{24}, e_{25}, e_{34}$, $\boldsymbol{e}_{35}, \boldsymbol{e}_{45}$

- ten trivectors: $e_{123}, e_{124}, e_{125}, e_{134}, e_{135}, e_{145}$, $\boldsymbol{e}_{234}, \boldsymbol{e}_{235}, \boldsymbol{e}_{245}, \boldsymbol{e}_{345}$

- five tetravectors: $e_{1234}, e_{1235}, e_{1245}, e_{1345}, e_{2345}$;

- one pseudoscalar: $\boldsymbol{e}_{12345}$.

For short, the product of the basis vectors $\boldsymbol{e}_{i}$ was denoted as $\boldsymbol{e}_{12} \equiv \boldsymbol{e}_{1} \boldsymbol{e}_{2}, \boldsymbol{e}_{123} \equiv \boldsymbol{e}_{1} \boldsymbol{e}_{2} \boldsymbol{e}_{3}$ etc. The vectors $\boldsymbol{e}_{i}$ span the five-dimensional real Euclidean space $\mathbb{R}^{5}$ having the positive-definite signature, i.e. the squares of basis vectors satisfy the relations $\boldsymbol{e}_{i}^{2}=+1$ for all $i$, and $\boldsymbol{e}_{i} \boldsymbol{e}_{j}=-\boldsymbol{e}_{j} \boldsymbol{e}_{i}$ if $i \neq j$. Both rules can be combined into the anticommutation relation

$$
\boldsymbol{e}_{i} \boldsymbol{e}_{j}+\boldsymbol{e}_{j} \boldsymbol{e}_{i}=2 \delta_{i j}
$$

where $\delta_{i j}$ is the Kronecker delta. Due to property (1), 
Clifford algebra elements make up a closed system, since the elements with repeated indices can be contracted, for example, $\boldsymbol{e}_{1232}=\boldsymbol{e}_{1} \boldsymbol{e}_{2} \boldsymbol{e}_{3} \boldsymbol{e}_{2}=-\boldsymbol{e}_{1} \boldsymbol{e}_{2} \boldsymbol{e}_{2} \boldsymbol{e}_{3}=-\boldsymbol{e}_{1} \boldsymbol{e}_{3}=$ $-\boldsymbol{e}_{13}$. With the help of contraction it can be shown that the squares of all bivectors and trivectors give minus one, $\boldsymbol{e}_{i j}^{2}=-1$ and $\boldsymbol{e}_{i j k}^{2}=-1$ (this is reminiscent of the imaginary unit i) and therefore are related with rotators in $\mathbb{R}^{5}$ space, while the squares of scalar, tetravectors and pseudoscalar $\boldsymbol{e}_{12345}$ give +1 .

The general vector $a$ in $\mathbb{R}^{5}$ can be expressed as a sum $a=\sum_{i} a_{i} \boldsymbol{e}_{i}$, where $a_{i}$ are real-valued components, $a_{i} \in \mathbb{R}$. The Clifford (or geometric) product of two vectors $a$ and $b$ can be decomposed into two parts, $a b=a \cdot b+a \wedge b$, where $a \cdot b$ is the scalar product and $a \wedge b$ is the wedge product. The latter geometrically represents an orientated plane. The scalar product is symmetric with respect to vector swapping, i.e. $a \cdot b=b \cdot a$, while the wedge product is antisymmetric, $a \wedge b=-b \wedge a$. Similarly, a general bivector can be written as a sum of elementary bivectors, $B=\sum_{i \neq j} b_{i j} \boldsymbol{e}_{i} \boldsymbol{e}_{j}$, where $b_{i j} \in \mathbb{R}$. The product of the vector $a$ and bivector $B$ brings about higher order wedge products of type $\boldsymbol{e}_{i} \wedge \boldsymbol{e}_{j} \wedge \boldsymbol{e}_{k}$, which represents an orientated $3 \mathrm{D}$ volume element. The highest order orientated elementary volume in $\mathbb{R}^{5}$ is given by $\boldsymbol{e}_{1} \wedge \boldsymbol{e}_{2} \wedge \boldsymbol{e}_{3} \wedge \boldsymbol{e}_{4} \wedge \boldsymbol{e}_{5}$. The wedged objects are called the blades. They represent elementary cells in $C l_{5}$. The important property is that under vector rotations in $\mathbb{R}^{n}$ space all blades transform with the same law.

A general multivector $M$ consists of a sum of multivectors of specific ranks

$$
M=\sum_{i=0}^{5}\langle M\rangle_{i}
$$

where $\langle M\rangle_{0}$ is the scalar part of the multivector, $\langle M\rangle_{1}$ is the vector part, $\langle M\rangle_{2}$ is the bivector part, etc. The index $i$ is called the grade. One can multiply the multivectors since the Clifford algebra, due to property (1), always remains closed. The product of multivectors is associative, $A(B C)=(A B) C=A B C$, however, it is noncommutative, $A B \neq B A$.

There is a number of operations over the multivectors in the Clifford algebra which we shall make use of. The reversion brings the basis vectors to reverse order and plays similar role as complex conjugation in the standard quantum mechanics. For example, the element $\boldsymbol{e}_{123}$ after the reversion becomes $\boldsymbol{e}_{321}$. The reversion will be denoted by tilde, thus, we shall write $\tilde{\boldsymbol{e}}_{123}=\boldsymbol{e}_{321}=-\boldsymbol{e}_{123}$, $\tilde{\boldsymbol{e}}_{1234}=\boldsymbol{e}_{4321}=\boldsymbol{e}_{1234}$. After the reversion the multivector (2) becomes

$$
\tilde{M}=\langle M\rangle_{0}+\langle M\rangle_{1}-\langle M\rangle_{2}-\langle M\rangle_{3}+\langle M\rangle_{4}+\langle M\rangle_{5} .
$$

If $C$ and $D$ are two multivectors, the reversion operation obeys the following rules: $(C D)^{-}=\tilde{D} \tilde{C},(C+D)^{\sim}=\tilde{C}+\tilde{D}$.

The other important operation is the exponentiation of the multivector that allows to rotate the vectors in $5 \mathrm{D}$ space. The exponential of the multivector $A$ is defined as a series:

$$
\mathrm{e}^{\mathrm{A}}=1+\frac{A}{1 !}+\frac{A^{2}}{2 !}+\frac{A^{3}}{3 !}+\ldots
$$

For a general multivector this series cannot be summed up in a closed form, at least, no such formula is known to present author. However, for specific multivectors the sum may be reduced to trigonometric or hyperbolic functions. For example, if the multivector consists of a scalar $s$ and vector $v=v_{1} \boldsymbol{e}_{1}+v_{2} \boldsymbol{e}_{2}+v_{3} \boldsymbol{e}_{3}+v_{4} \boldsymbol{e}_{4}+v_{5} \boldsymbol{e}_{5}$, the exponential gives

$$
\mathrm{e}^{s+v}=\mathrm{e}^{s}\left(\sinh \sqrt{v \tilde{v}}+\frac{v}{\sqrt{v \tilde{v}}} \cosh \sqrt{v \tilde{v}}\right),
$$

where $v \tilde{v}=v_{1}^{2}+v_{2}^{2}+v_{3}^{2}+v_{4}^{2}+v_{5}^{2}$.

Similar situation holds for scalar and tetravector $w=$ $w_{1} \boldsymbol{e}_{1234}+w_{2} \boldsymbol{e}_{1235}+w_{3} \boldsymbol{e}_{1245}+w_{4} \boldsymbol{e}_{1345}+w_{5} \boldsymbol{e}_{2345}$,

$$
\mathrm{e}^{s+w}=\mathrm{e}^{s}\left(\sinh \sqrt{w \tilde{w}}+\frac{w}{\sqrt{w \tilde{w}}} \cosh \sqrt{w \tilde{w}}\right),
$$

where $w \tilde{w}=w_{1}^{2}+w_{2}^{2}+w_{3}^{2}+w_{4}^{2}+w_{5}^{2}$.

The general bivector $B$ consists of ten terms: $B=$ $b_{1} \boldsymbol{e}_{1} \boldsymbol{e}_{2}+b_{2} \boldsymbol{e}_{1} \boldsymbol{e}_{3}+b_{3} \boldsymbol{e}_{1} \boldsymbol{e}_{4}+b_{4} \boldsymbol{e}_{1} \boldsymbol{e}_{5}+b_{5} \boldsymbol{e}_{2} \boldsymbol{e}_{3}+b_{6} \boldsymbol{e}_{2} \boldsymbol{e}_{4}+$ $b_{7} \boldsymbol{e}_{2} \boldsymbol{e}_{5}+b_{8} \boldsymbol{e}_{3} \boldsymbol{e}_{4}+b_{9} \boldsymbol{e}_{3} \boldsymbol{e}_{5}+b_{10} \boldsymbol{e}_{4} \boldsymbol{e}_{5}$. Since, in general, the series of $\mathrm{e}^{B}$ cannot be summed up, only special cases will be considered that will suffice for current calculations. The summation of the bivector exponential series is based on the following observation: the fourth power of the bivector $B$ can be expressed through the scalar part of $B \tilde{B}$,

$$
\langle B \tilde{B}\rangle_{0}=\sum_{i=1}^{10} b_{i}^{2}
$$

and the square of $B$. Namely, we find that

$$
B^{4}=\left\langle B^{4}\right\rangle_{0}-2\langle B \tilde{B}\rangle_{0}^{2}-2\langle B \tilde{B}\rangle_{0} B^{2}
$$

for a general $B$. This equation is nothing else but a recurrence formula that allows to express higher powers in the series (4) by lower powers of $B$. However, the resulting series still appears too complicated to be summed up. To introduce some simplifications, the recurrence relation (8) will be rewritten as a product of two perpendicular multivectors

$$
\left(B^{2}+\langle B \tilde{B}\rangle_{0}+\alpha^{2}\right)\left(B^{2}+\langle B \tilde{B}\rangle_{0}-\alpha^{2}\right)=0,
$$

where $\alpha^{2}=\sqrt{\left\langle B^{4}\right\rangle_{0}-\langle B \tilde{B}\rangle_{0}^{2}}$. It should be noted that the individual factors in the parentheses of Eq. (9) cannot be equated to zero. Only their product gives zero. If $\alpha=0$, the multivector factors in (9) become parallel, which is equivalent to condition $\left\langle B^{4}\right\rangle_{0}=\langle B \tilde{B}\rangle_{0}^{2}$, i.e. the scalar part of the fourth power of the multivector becomes equal to square of the bivector magnitude $\langle B \tilde{B}\rangle_{0}$. If this does happen then it follows from Eq. (9) that the square of the bivector reduces to the scalar

$$
B^{2}=-\langle B \tilde{B}\rangle_{0} \text {. }
$$

Under these conditions the exponential can be summed up to

$$
\mathrm{e}^{B}=\cos \sqrt{\langle B \tilde{B}\rangle_{0}}+\frac{B}{\sqrt{\langle B \tilde{B}\rangle_{0}}} \sin \sqrt{\langle B \tilde{B}\rangle_{0}} .
$$

The minus sign in (10) guarantees that now the trigono- 
metric functions appear instead of hyperbolic ones. The condition (10), as can be seen from recurrence Eq. (8), is equivalent to $B^{4}=\left\langle B^{4}\right\rangle_{0}$, i.e. the fourth power of the bivector should give the scalar. For example, the bivector $B_{1}=b_{1} \boldsymbol{e}_{12}+b_{2} \boldsymbol{e}_{13}+b_{3} \boldsymbol{e}_{14}+b_{4} \boldsymbol{e}_{15}$ satisfies the mentioned condition (10): $B_{1}^{2}=-\left(b_{1}^{2}+b_{2}^{2}+b_{3}^{2}+b_{4}^{2}\right)$. The bivector $B_{2}=b_{1} \boldsymbol{e}_{12}+b_{5} \boldsymbol{e}_{23}+b_{6} \boldsymbol{e}_{24}+b_{7} \boldsymbol{e}_{25}$ also satisfies this condition: $B_{2}^{2}=-\left(b_{1}^{2}+b_{5}^{2}+b_{6}^{2}+b_{7}^{2}\right)$.

\subsection{Representation of basis vectors}

In the literature, the most widely used representation of the basis vectors employs the square matrices. The algebra $C l_{5}$ is isomorphic to $2 \times 2$ matrices the elements of which are quaternions. In particular, the following correspondence exists [2]:

$$
\begin{aligned}
& \boldsymbol{e}_{1}=\left[\begin{array}{cc}
0 & -\mathrm{i} \\
\mathrm{i} & 0
\end{array}\right], \quad \boldsymbol{e}_{2}=\left[\begin{array}{cc}
0 & -\mathrm{j} \\
\mathrm{j} & 0
\end{array}\right], \\
& \boldsymbol{e}_{3}=\left[\begin{array}{cc}
0 & -\mathrm{k} \\
\mathrm{k} & 0
\end{array}\right], \\
& \boldsymbol{e}_{4}=\left[\begin{array}{cc}
1 & 0 \\
0 & -1
\end{array}\right], \quad \boldsymbol{e}_{5}=\left[\begin{array}{ll}
0 & 1 \\
1 & 0
\end{array}\right] .
\end{aligned}
$$

Here $\mathrm{i}, \mathrm{j}$, and $\mathrm{k}$ are quaternionic imaginary units with properties $\mathrm{i}^{2}=\mathrm{j}^{2}=\mathrm{k}^{2}=\mathrm{ijk}=-1$. However, this quaternionic representation is not suitable for our purpose since the objects we would like to transform to the Clifford algebra are complex $4 \times 4$ Hermitian matrices. The required Dirac-like matrices having positive signature $(+,+,+,+,+)$ were given in $[11,12]$. As we shall see, the following set of $\Gamma_{i}$ matrices yields real coefficients at basis vectors $\boldsymbol{e}_{i}$, as required by $C l_{5}$ after the decomposition of the Hamiltonian and hole spin matrices,

$$
\begin{aligned}
& \boldsymbol{e}_{1} \rightarrow \Gamma_{1}=\sigma_{z} \otimes \sigma_{y}=\left[\begin{array}{cccc}
0 & -\mathrm{i} & 0 & 0 \\
\mathrm{i} & 0 & 0 & 0 \\
0 & 0 & 0 & \mathrm{i} \\
0 & 0 & -\mathrm{i} & 0
\end{array}\right], \\
& \boldsymbol{e}_{2} \rightarrow \Gamma_{2}=\sigma_{z} \otimes \sigma_{x}=\left[\begin{array}{cccc}
0 & 1 & 0 & 0 \\
1 & 0 & 0 & 0 \\
0 & 0 & 0 & -1 \\
0 & 0 & -1 & 0
\end{array}\right], \\
& \boldsymbol{e}_{3} \rightarrow \Gamma_{3}=\sigma_{y} \otimes 1= \\
& \boldsymbol{e}_{4} \rightarrow \Gamma_{4}=\sigma_{x} \otimes 1= \\
& 0
\end{aligned}
$$

$$
\boldsymbol{e}_{5} \rightarrow \Gamma_{5}=\sigma_{z} \otimes \sigma_{z}=\left[\begin{array}{cccc}
1 & 0 & 0 & 0 \\
0 & -1 & 0 & 0 \\
0 & 0 & -1 & 0 \\
0 & 0 & 0 & 1
\end{array}\right]
$$

These matrices satisfy the property $(1)$ :

$$
\Gamma_{i} \Gamma_{j}+\Gamma_{j} \Gamma_{i}=2 \delta_{i j},
$$

where $\delta_{i i}$ now is the unit matrix. The matrix representations of the bivectors $\boldsymbol{e}_{i j} \rightarrow \Gamma_{i j}$, trivectors $\boldsymbol{e}_{i j k} \rightarrow \Gamma_{i j k}$ etc. can be found by multiplying the respective matrices: $\Gamma_{i j}=\Gamma_{i} \Gamma_{j}, \Gamma_{i j k}=\Gamma_{i} \Gamma_{j} \Gamma_{k}$ etc. It should be noted that the matrix representation (14)-(18) is not isomorphic to $\mathrm{Cl}_{5}$ algebra. For example, it is easy to show that matrix representations of vectors and tetravectors are related in the following way: $\Gamma_{1}=-\Gamma_{2345}, \Gamma_{2}=\Gamma_{1345}$, $\Gamma_{3}=-\Gamma_{1245}, \Gamma_{4}=\Gamma_{1235}, \Gamma_{5}=-\Gamma_{1234}$. Apart from the mentioned vector-tetravector pair similarity there are similar equalities, with positive and negative signs, between bivector-trivector and scalar-pseudoscalar pairs. However, the matrix representation is homomorphic to $\mathrm{Cl}_{5}$ and, therefore, all group operations are preserved. To avoid misconstructions in the following we shall map the Hilbert space vectors and operators onto scalars, vectors and bivectors only.

With the matrix representation (14)-(18) known, one can decompose any complex matrix $m$ into sum of its constituent basis elements of $C l_{5}$. The required real-valued components (projections) $c_{b}$ can be found from

$$
c_{b}=\operatorname{Tr}\left(m \Gamma_{b}\right),
$$

where $\operatorname{Tr}$ is the trace and $b$ is one of indices in $\boldsymbol{e}_{0}, \boldsymbol{e}_{i}, \boldsymbol{e}_{i j}$.

\section{Valence band and spin in the Clifford algebra $\mathrm{Cl}_{5}$}

Within spherical energy band approximation the Luttinger-Kohn valence band Hamiltonian is [6]:

$$
H=\frac{1}{2 m_{0}}\left[\left(\gamma_{1}+\frac{5}{2} \gamma_{2}\right) \boldsymbol{k}^{2}-2 \gamma_{2}(\boldsymbol{k} \cdot \boldsymbol{J})^{2}\right],
$$

where $\gamma_{1}$ and $\gamma_{2}$ are band parameters, $m_{0}$ is the electron mass, and $\boldsymbol{k}=\left(k_{x}, k_{y}, k_{z}\right)$ is the hole wave vector. $\boldsymbol{J}=\left(J_{x}, J_{y}, J_{z}\right)$ is the vectorial $4 \times 4$ total angular momentum represented by the following matrices:

$$
\begin{aligned}
& J_{x}=\left[\begin{array}{cccc}
0 & \frac{\sqrt{3}}{2} & 0 & 0 \\
\frac{\sqrt{3}}{2} & 0 & 0 & 0 \\
0 & 0 & 0 & \frac{\sqrt{3}}{2} \\
0 & 0 & \frac{\sqrt{3}}{2} & 0
\end{array}\right], \\
& J_{y}=\left[\begin{array}{cccc}
0 & -\frac{\mathrm{i} \sqrt{3}}{2} & 0 & 0 \\
\frac{\mathrm{i} \sqrt{3}}{2} & 0 & 0 & 0 \\
0 & 0 & 0 & -\frac{\mathrm{i} \sqrt{3}}{2} \\
0 & 0 & \frac{\mathrm{i} \sqrt{3}}{2} & 0
\end{array}\right],
\end{aligned}
$$




$$
J_{z}=\left[\begin{array}{cccc}
\frac{3}{2} & 0 & 0 & 0 \\
0 & \frac{1}{2} & 0 & 0 \\
0 & 0 & -\frac{1}{2} & 0 \\
0 & 0 & 0 & -\frac{3}{2}
\end{array}\right] .
$$

In the considered approximation the spin operator $\boldsymbol{S}$ differs from $\boldsymbol{J}$ by a constant, $\boldsymbol{S}=\frac{1}{3} \boldsymbol{J}$. Therefore, we shall refer to $\boldsymbol{J}$ as the spin matrix, too.

With the help of (20) the Hamiltonian (21) can be rewritten in the $C l_{5}$ basis as

$$
H=\frac{\gamma_{1} k^{2}}{2 m_{0}}+\frac{\gamma_{2} k^{2}}{m_{0}} d, \quad d=\sum_{n=1}^{5} d_{n} \boldsymbol{e}_{n},
$$

where $k^{2}=k_{x}^{2}+k_{y}^{2}+k_{z}^{2}$ and $d_{n}$ are the real-valued projections on the respective basis vectors $\boldsymbol{e}_{i}$ in 5D Euclidean space

$$
\begin{aligned}
& d_{1}=-\sqrt{3} k_{y} k_{z} / k^{2}, \\
& d_{2}=-\sqrt{3} k_{x} k_{z} / k^{2}, \\
& d_{3}=-\sqrt{3} k_{x} k_{y} / k^{2}, \\
& d_{4}=-\frac{\sqrt{3}}{2}\left(k_{x}^{2}-k_{y}^{2}\right) / k^{2}, \\
& d_{5}=-\frac{1}{2}\left(2 k_{z}^{2}-k_{x}^{2}-k_{y}^{2}\right) / k^{2} .
\end{aligned}
$$

From (25) it is seen that the Hamiltonian is made up of the scalar and vector in $\mathbb{R}^{5}$. On the other hand, the decomposition of spin matrices (22)-(24) shows that they are made up of bivectors only

$$
\begin{aligned}
J_{x} & =\frac{\mathrm{i}}{2}\left(-e_{1} e_{4}-\sqrt{3} e_{1} e_{5}+e_{2} e_{3}\right), \\
J_{y} & =\frac{\mathrm{i}}{2}\left(-e_{1} e_{3}+\sqrt{3} e_{2} e_{5}-e_{2} e_{4}\right), \\
J_{z} & =\frac{\mathrm{i}}{2}\left(\boldsymbol{e}_{1} \boldsymbol{e}_{2}+2 \boldsymbol{e}_{3} \boldsymbol{e}_{4}\right) .
\end{aligned}
$$

This is consistent with the fact that the bivectors in the Clifford algebra are related with rotations in various planes. Using the anticommutation relation (1) it is easy to show that the operators (31)-(33) satisfy the conservation law for quantum number $j=3 / 2$,

$$
J_{x}^{2}+J_{y}^{2}+J_{z}^{2}=j(j+1)=15 / 4
$$

New operators (25), (31)-(33) act in the Clifford space where the operators and multivectors reside, while the old ones (21)-(24) act on the spinors in the Hilbert space.

\subsection{Rotation of basis vectors}

One of the most useful operations in the Clifford algebra is the rotation of $5 \mathrm{D}$ vector in $\mathbb{R}^{5}$ space spanned by $\boldsymbol{e}_{1}, \ldots, \boldsymbol{e}_{5}$ basis. We shall show that a proper selection of the rotations allows one to find eigenvalues of the Hamiltonian.

The rotation by angle $\theta$ in the plane spanned by bivector $\boldsymbol{e}_{i} \boldsymbol{e}_{j}$ is performed with the help of a rotor

$$
R_{i j}(\theta / 2)=\mathrm{e}^{\frac{\theta}{2} \boldsymbol{e}_{i} \boldsymbol{e}_{j}}=\cos (\theta / 2)+\boldsymbol{e}_{i} \boldsymbol{e}_{j} \sin (\theta / 2)
$$

and its reverse $\tilde{R}_{i j}(\theta / 2)[2,4]$. The last expression follows from Eq. (11). The rotor $R$, in general, satisfies the condition $R \tilde{R}=\tilde{R} R=1$. For example, the rotation of the vector $\boldsymbol{e}_{1}$ by an angle $\varphi$ in bivector plane $\boldsymbol{e}_{1} \boldsymbol{e}_{2}$ gives new vector

$$
\begin{aligned}
\boldsymbol{e}_{1}^{\prime} & =\tilde{R}_{12}(\varphi / 2) \boldsymbol{e}_{1} R_{12}(\varphi / 2)=\mathrm{e}^{\frac{\varphi}{2}} \boldsymbol{e}_{2} \boldsymbol{e}_{1} \boldsymbol{e}_{1} \mathrm{e}^{\frac{\varphi}{2} \boldsymbol{e}_{1} \boldsymbol{e}_{2}} \\
& =\boldsymbol{e}_{1} \cos \varphi+\boldsymbol{e}_{2} \sin \varphi .
\end{aligned}
$$

However, the rotation of the same vector $\boldsymbol{e}_{1}$ in a plane that does not hold the vector, for example $\boldsymbol{e}_{3} \boldsymbol{e}_{5}$, gives no rotation at all,

$$
\boldsymbol{e}_{1}^{\prime \prime}=\tilde{R}_{35}(\varphi / 2) \boldsymbol{e}_{1} R_{35}(\varphi / 2)=\boldsymbol{e}_{1} .
$$

This property is characteristic of spaces higher than $3 \mathrm{D}$, since in the higher order spaces nonparallel planes do not necessarily intersect as it is in $3 \mathrm{D}$.

Before going to eigenvalues of the Hamiltonian, it should be noted that at $k_{x}=k_{y}=0$ the initial Hamiltonian is diagonal and the vector $d$ in (25) becomes parallel to $\boldsymbol{e}_{5}$. This suggests that the rotation of $d$ in $\mathbb{R}^{5}$ space must end up in $\boldsymbol{e}_{5}$ direction. On the other hand, Eqs. (36) and (37) suggest that for all possible rotations to take place the bivector planes should be mutually coupled. From this follows that the rotor that "diagonalizes" the Hamiltonian (25) should have the following structure:

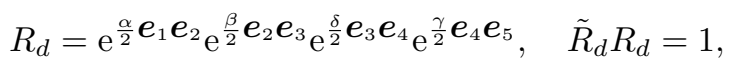

where the angles $\alpha, \beta, \delta$ and $\gamma$ are to be determined. If the rotor (38) is applied to individual basis vectors, $\boldsymbol{e}_{i}^{\prime}=\tilde{R}_{d} \boldsymbol{e}_{i} R_{d}$, the new vectors are found to be related to old ones in the following way

$$
\begin{aligned}
\boldsymbol{e}_{5}^{\prime} & =-\boldsymbol{e}_{4} s_{\alpha}+\boldsymbol{e}_{5} c_{\alpha}, \\
\boldsymbol{e}_{4}^{\prime} & =-\boldsymbol{e}_{3} s_{\beta}+\boldsymbol{e}_{4} c_{\alpha} c_{\beta}+\boldsymbol{e}_{5} s_{\alpha} c_{\beta}, \\
\boldsymbol{e}_{3}^{\prime} & =-\boldsymbol{e}_{2} s_{\delta}+\boldsymbol{e}_{3} c_{\beta} c_{\delta}+\boldsymbol{e}_{4} c_{\alpha} s_{\beta} c_{\delta}+\boldsymbol{e}_{5} s_{\alpha} s_{\beta} c_{\delta}, \\
\boldsymbol{e}_{2}^{\prime} & =-\boldsymbol{e}_{1} s_{\gamma}+\boldsymbol{e}_{2} c_{\delta} c_{\gamma}+\boldsymbol{e}_{3} c_{\beta} s_{\delta} c_{\gamma}+\boldsymbol{e}_{4} c_{\alpha} s_{\beta} s_{\delta} c_{\gamma} \\
& +\boldsymbol{e}_{5} s_{\alpha} s_{\beta} s_{\delta} c_{\gamma}, \\
\boldsymbol{e}_{1}^{\prime} & =+\boldsymbol{e}_{1} c_{\gamma}+\boldsymbol{e}_{2} c_{\delta} s_{\gamma}+\boldsymbol{e}_{3} c_{\beta} s_{\delta} s_{\gamma}+\boldsymbol{e}_{4} c_{\alpha} s_{\beta} s_{\delta} s_{\gamma} \\
& +\boldsymbol{e}_{5} s_{\alpha} s_{\beta} s_{\delta} s_{\gamma},
\end{aligned}
$$

where the following shorthands $s_{\alpha}=\sin \alpha, c_{\alpha}=\cos \alpha$, etc. were introduced.

To connect the components of the wave vector $\boldsymbol{k}$ in 3D space with the rotation angles in $5 \mathrm{D}$ space the components of a general vector $a$,

$$
\begin{aligned}
a & =R_{a} \boldsymbol{e}_{5} \tilde{R}_{a}=\boldsymbol{e}_{1} \sin \alpha \sin \beta \sin \delta \sin \gamma \\
& +\boldsymbol{e}_{2} \sin \alpha \sin \beta \sin \delta \cos \gamma+\boldsymbol{e}_{3} \sin \alpha \sin \beta \cos \delta \\
& +\boldsymbol{e}_{4} \sin \alpha \cos \beta+\boldsymbol{e}_{5} \cos \alpha,
\end{aligned}
$$

will be equated to the components (26)-(30). Since at $k_{x}=k_{y}=0$ and $\alpha=0$ one has, respectively, $d=(0,0,0,0,-1)$ and $a=e_{5}$, it is convenient to fix the angle $\alpha$ by $a_{5} \equiv \cos \alpha=\left(k_{x}^{2}+k_{y}^{2}-2 k_{z}^{2}\right) / 2 k^{2}$. The pairing of remaining $d_{i}$ and $a_{i}$ components remains arbitrary. In the following we shall equate the components in the same order as they are given in Eqs. (44) and (26)-(30), i.e. we shall assume that $d_{i}=a_{i}$, where $a_{i}$ are the components of the vector $a$ in (44). The solution of the resulting system 
of equations $d_{i}=a_{i}$ with $i=1, \ldots, 5$, yields

$$
\begin{aligned}
& \cos \alpha=d_{5}, \\
& \cos \beta=d_{4} / \sqrt{1-d_{5}^{2}}, \\
& \cos \delta=d_{3} / \sqrt{1-d_{4}^{2}-d_{5}^{2}}, \\
& \cos \gamma=d_{2} / \sqrt{1-d_{3}^{2}-d_{4}^{2}-d_{5}^{2}} .
\end{aligned}
$$

The equation for $d_{1}$ represents radius of $4 \mathrm{D}$ sphere, $d_{1}^{2}+$ $d_{2}^{2}+d_{3}^{2}+d_{4}^{2}+d_{5}^{2}=1$.

The eigenvalues of the Hamiltonian (25) are calculated with the constructed rotor $R_{d}$ that transforms the basis vectors to new ones given by (39)-(43). Then, after insertion of (45)-(48) and (26)-(30) one finds

$$
\begin{aligned}
H_{d} & =\tilde{R}_{d} H R_{d}=\frac{k^{2}}{2 m_{0}}\left(\gamma_{1}+2 \gamma_{2} \boldsymbol{e}_{5}\right) \\
\rightarrow & {\left[\begin{array}{cccc}
\varepsilon_{\mathrm{L}} & 0 & 0 & 0 \\
0 & \varepsilon_{\mathrm{H}} & 0 & 0 \\
0 & 0 & \varepsilon_{\mathrm{H}} & 0 \\
0 & 0 & 0 & \varepsilon_{\mathrm{L}}
\end{array}\right] }
\end{aligned}
$$

The matrix representation (18) was used to translate the Hamiltonian to matrix form. The diagonal terms in this matrix are dispersions of heavy- and light-mass energy bands of holes,

$$
\varepsilon_{\mathrm{H}, \mathrm{L}}=\frac{\gamma_{1} \pm 2 \gamma_{2}}{2 m_{0}} k^{2}
$$

where the minus and plus correspond, respectively, to $\mathrm{H}$ and L bands.

\subsection{Spin surface}

The energy bands are doubly degenerate and, in the Hilbert space, they are described by four-component spinors. The spin polarization depends on a relative magnitude and phase between spinor components. In the Clifford algebra approach the hole spin properties are determined by coefficients at bivectors in equations of type (31)-(33), where the basis vectors $\boldsymbol{e}_{i}$ should be replaced by transformed ones (39)-(43). The resulting new polarization vector $\boldsymbol{J}^{\prime}=\left(J_{x}^{\prime}, J_{x}^{\prime}, J_{x}^{\prime}\right)$ then will be in the energy representation. As an illustration we shall calculate lightand heavy-hole spin surfaces.

The spin surface represents the loci of the ends of all possible spin polarization vectors [13]. In case of conduction band electron the spin surface is represented by the Bloch sphere. It can be calculated after parametrization of light- and heavy-hole spinors by two parameters $\vartheta$ and $\phi$ in the energy representation for a given hole wave vector $\boldsymbol{k}$,

$$
\begin{aligned}
& \psi_{\mathrm{L}}=\left(\cos (\vartheta / 2) \mathrm{e}^{-\mathrm{i} \phi / 2}, 0,0, \sin (\vartheta / 2) \mathrm{e}^{\mathrm{i} \phi / 2}\right), \\
& \psi_{\mathrm{H}}=\left(0, \cos (\vartheta / 2) \mathrm{e}^{-\mathrm{i} \phi / 2}, \sin (\vartheta / 2) \mathrm{e}^{\mathrm{i} \phi / 2}, 0\right) .
\end{aligned}
$$

Then, the average spin polarization vector will be

$$
\langle\boldsymbol{J}\rangle_{\mathrm{L}, \mathrm{H}}=\left\langle\boldsymbol{J}^{\prime}\right\rangle_{\mathrm{L}, \mathrm{H}}=\left\langle\psi_{\mathrm{L}, \mathrm{H}}\left|\boldsymbol{J}^{\prime}\right| \psi_{\mathrm{L}, \mathrm{H}}\right\rangle,
$$

mentum operator [see Eqs. (31)-(33)] the vectors $\boldsymbol{e}_{i}$ should be replaced by primed ones given by Eqs. (39)(43) and which correspond to diagonal Hamiltonian. To come back to the Hilbert space it is enough to replace the bivectors $\boldsymbol{e}_{i} \boldsymbol{e}_{j}$ by respective matrix products $\Gamma_{i} \Gamma_{j}$. The calculations give the following heavy-mass hole polarization vector:

$$
\langle\boldsymbol{J}\rangle_{\mathrm{H}}=\left(k_{x}, k_{y}, k_{z}\right) Q,
$$

where

$$
\begin{aligned}
Q & =3\left[k_{x} k_{y} k_{z} p \cos \vartheta+\left(\left(k^{2}+k_{z}^{2}\right) s^{2} \cos \phi\right.\right. \\
& \left.\left.+\left(k_{x}^{2}-k_{y}^{2}\right) k_{z}^{2} \sin \phi\right) \sin \vartheta\right] /\left(2 p k^{2} s^{2}\right) .
\end{aligned}
$$

Here $p^{2}=k^{2}+3 k_{z}^{2}, s^{2}=\sqrt{k_{x}^{2} k_{y}^{2}+k_{x}^{2} k_{z}^{2}+k_{y}^{2} k_{z}^{2}}$.

From Eq. (54) follows that the heavy-hole spin surface reduces to line parallel to $\boldsymbol{k}$. For $\boldsymbol{k}=\left(k_{x}, 0,0\right), \boldsymbol{k}=$ $\left(0, k_{y}, 0\right)$, and $\boldsymbol{k}=\left(0,0, k_{z}\right)$ Eq. (54) gives, respectively,

$$
\begin{aligned}
\langle\boldsymbol{J}\rangle_{\mathrm{H} x} & =\left(\frac{3}{2} \sin \vartheta \cos \phi, 0,0\right), \\
\langle\boldsymbol{J}\rangle_{\mathrm{H} y} & =\left(0, \frac{3}{2} \sin \vartheta \cos \phi, 0\right), \\
\langle\boldsymbol{J}\rangle_{\mathrm{H} z} & =\left(0,0, \frac{3}{2} \sin \vartheta \cos \phi\right) .
\end{aligned}
$$

Thus, the heavy-hole average spin cannot be represented on the Bloch sphere. The polarization vector can assume any value, including zero, between $\pm 3 / 2$ and always is parallel to $\boldsymbol{k}$. The analysis of (54) shows that this property holds for any wave vector $\boldsymbol{k}$.

For a light-hole the average polarization vector was found to be more complicated

$$
\begin{aligned}
& \langle\boldsymbol{J}\rangle_{\mathrm{L}}=\left\langle\boldsymbol{J}^{\prime}\right\rangle_{\mathrm{L}}=\left(\left\langle J_{x}^{\prime}\right\rangle,\left\langle J_{y}^{\prime}\right\rangle,\left\langle J_{z}^{\prime}\right\rangle\right)_{\mathrm{L}} \\
& =\left(j_{x}^{\prime}, j_{y}^{\prime}, j_{z}^{\prime}\right) /\left(2 p k^{2} s^{2}\right),
\end{aligned}
$$

where

$$
\begin{aligned}
& j_{x}^{\prime}=-k_{y} k_{z} p\left(2 k^{2}-3 k_{x}^{2}\right) \cos \vartheta \\
& \quad-k_{x} p\left(s^{2} \cos \phi+\left(2 k_{y}^{2}+k_{z}^{2}\right) \sin \phi\right) \sin \vartheta, \\
& j_{y}^{\prime}=-k_{z} k_{x} p\left(2 k^{2}-3 k_{y}^{2}\right) \cos \vartheta \\
& \quad-k_{y} p\left(s^{2} \cos \phi-\left(2 k_{x}^{2}+k_{z}^{2}\right) \sin \phi\right) \sin \vartheta, \\
& j_{z}^{\prime}=-k_{x} k_{y} p\left(2 k^{2}-3 k_{z}^{2}\right) \cos \vartheta \\
& \quad+k_{z}\left[\left(5 k^{2}-3 k_{z}^{2}\right) s^{2} \cos \phi\right. \\
& \left.\quad-\left(2 k_{x}^{4}-2 k_{y}^{4}-k_{x}^{2} k_{z}^{2}+k_{y}^{2} k_{z}^{2}\right) \sin \phi\right] \sin \vartheta .
\end{aligned}
$$

If the wave vector is directed along principle crystallographic axes, i.e. $\boldsymbol{k}=\left(k_{x}, 0,0\right), \boldsymbol{k}=\left(0, k_{y}, 0\right)$, or $\boldsymbol{k}=\left(0,0, k_{z}\right)$, the formulae (59)-(62) simplify, respectively, to

$$
\langle\boldsymbol{J}\rangle_{\mathrm{L} x}=\left(-\frac{1}{2} \sin \vartheta \cos \phi, \sin \vartheta \sin \phi,-\cos \vartheta\right),
$$




$$
\begin{aligned}
\langle\boldsymbol{J}\rangle_{\mathrm{L} y} & =\left(-\sin \vartheta \sin \phi,-\frac{1}{2} \sin \vartheta \cos \phi,-\cos \vartheta\right), \\
\langle\boldsymbol{J}\rangle_{\mathrm{L} z} & =\left(-\cos \vartheta, \sin \vartheta \sin \phi, \frac{1}{2} \sin \vartheta \cos \phi\right) .
\end{aligned}
$$

Figure 1 depicts the spin surface $\langle\boldsymbol{J}\rangle_{\mathrm{L} z}$ along with that for $\langle\boldsymbol{J}\rangle_{\mathrm{H} z}$. It should be stressed once more that only the shape of the surface matters rather than a concrete formula that represents a grid, which merely visualizes the surface. By this reason, depending on the method of calculation, one can get different formulae for the same spin surface. For example Eq. (65) may also be written in the form

$$
\langle\boldsymbol{J}\rangle_{\mathrm{L} z}=\left(-\sin \vartheta \sin \phi,-\cos \vartheta, \frac{1}{2} \sin \vartheta \cos \phi\right) .
$$

However the orientation of the surface depends on direction of $\boldsymbol{k}$. This is seen in Eqs. (63)-(65) as well.

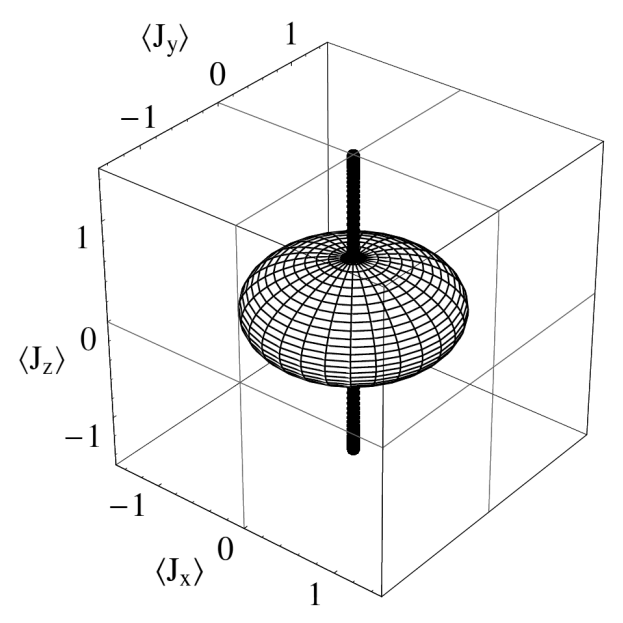

Fig. 1. Spin surfaces of heavy-mass (vertical line) and light-mass (oblate spheroid) holes when $\boldsymbol{k} \|[001]$. For other directions of $\boldsymbol{k}$ the spin surfaces should be rotated in the same way as $\boldsymbol{k}$.

Finally, the following point will be noted. In the general equation (59) the polarization components appear in a form $(a \sin \vartheta \cos \phi, b \sin \vartheta \sin \phi, c \cos \vartheta)$, which suggests that they represent three perpendicular vectors $\boldsymbol{u}, \boldsymbol{v}$ and $\boldsymbol{w}$ having polar components $(a, b, c)$. It can be shown that the volume spanned by the vectors $\boldsymbol{u}=\left\langle J_{x}^{\prime}\right\rangle_{\mathrm{L}}, \boldsymbol{v}=\left\langle J_{y}^{\prime}\right\rangle_{\mathrm{L}}$, and $\boldsymbol{w}=\left\langle J_{z}^{\prime}\right\rangle_{\mathrm{L}}$ is $\boldsymbol{u} \cdot \boldsymbol{v} \times \boldsymbol{w}=1 / 2$, which is independent of $\boldsymbol{k}$ and spinor parametrization scheme, and, apart from a multiplicative constant, gives the volume enclosed by light-hole spin surface. Thus, we conclude that the volume enclosed by the spin surface represents the invariant within the spherical energy band approximation.

\section{Summary}

In the standard quantum mechanics the diagonalization of the operators that act on the Hilbert space spinors is done with an appropriate unitary transformation. The present paper shows that in the Clifford algebra this can be achieved with rotation of vectors in Euclidean $\mathbb{R}^{n}$ space. Also the paper demonstrates how this can be achieved in spherical and doubly degenerate valence band case. In particular, it is shown that the Hamiltonian can be diagonalized with four rotations of $5 \mathrm{D}$ vector in coupled elementary bivector planes. In the Clifford algebra the borderline between operators and spinors vanishes and both are treated on equal footing with the help of multivectors that are made up of products of basis vectors $\boldsymbol{e}_{i}$. Thus, the rotation of basis vectors in the Euclidean space automatically is reflected in the structure of all Clifford operators, including spin or total angular momentum operators. This was demonstrated by calculating the spin surfaces of light- and heavy-holes, which appeared to be invariants within the approximation used in this paper.

\section{Acknowledgments}

The work was partly supported by Lithuanian State Science and Studies Foundation under contract C-07004.

\section{References}

[1] D. Hestenes, G. Sobczyk, Clifford Algebra to Geometric Calculus, Reidel, Boston 1984.

[2] P. Lounesto, Clifford Algebras and Spinors, Cambridge University Press, Cambridge 1997.

[3] D. Hestenes, Am. J. Phys. 71, 104 (2003).

[4] C. Doran, A. Lasenby, Geometric Algebra for Physicists, Cambridge University Press, Cambridge 2003.

[5] C. Doran, A. Lasenby, S. Gull, S. Somaroo, A. Challinor, arXiv:quant-ph/0509178v1, 1-93 (2005).

[6] J.M. Luttinger, W. Kohn, Phys. Rev. 97, 869 (1955).

[7] A. Dargys, Phys. Status Solidi B 241, 2954 (2004).

[8] A. Dargys, Solid-State Electron. 51, 93 (2007).

[9] A. Dargys, Phys. Status Solidi B 245, 1483 (2008).

[10] A. Dargys, Phys. Scr. 79, 055702 (2009).

[11] E. Demler, S.C. Zhang, Ann. Phys. 271, 83 (1999).

[12] S. Murakami, N. Nagaosa, S.C. Zhang, Phys. Rev. B 69, 235206 (2004).

[13] A. Dargys, in: Spintronics: Materials, Applications and Devices, Eds. G.C. Lombardi, G.E. Bianchi, NOVA Publishers, New York 2008. 